\title{
Oculomotor inhibition of return in normal and mindless reading
}

\author{
John M. Henderson • Steven G. Luke
}

Published online: 2 October 2012

(C) Psychonomic Society, Inc. 2012

\begin{abstract}
Oculomotor inhibition of return (O-IOR) is an increase in saccade latency prior to an eye movement to a recently fixated location, as compared with other locations. To investigate O-IOR in reading, subjects participated in two conditions while their eye movements were recorded: normal reading and mindless reading with words replaced by geometric shapes. We investigated the manifestation of $\mathrm{O}-\mathrm{IOR}$ in reading and whether it is related to extracting meaning from the text or is an oculomotor phenomenon. The results indicated that fixation durations prior to a saccade returning to the immediately preceding fixated word were longer than those to other words, consistent with O-IOR. Furthermore, fixation durations were longest prior to a saccade that returned the eyes to the specific character position in the word that had previously been fixated and dropped off as the distance between the previously fixated character and landing position increased. This result is consistent with the hypothesis that O-IOR is relatively precise in its application during reading and drops off as a gradient. Both of these results were found for text reading and for mindless reading, suggesting that they are consequences of oculomotor control, and not of language processing. Finally, although these temporal IOR effects were robust, no spatial consequences of IOR were observed: Previously fixated words and characters were as likely to be refixated as new words and characters.
\end{abstract}

\footnotetext{
J. M. Henderson • S. G. Luke

University of South Carolina,

Columbia, USA

J. M. Henderson $(\square)$

Department of Psychology and McCausland Center for Brain

Imaging, University of South Carolina,

Columbia, SC 29208, USA

e-mail: john.henderson@sc.edu
}

Keywords Eye movements and visual attention · Eye movements and reading $\cdot$ Inhibition of return

During reading, the eyes move through text in a series of fixations and saccades (Rayner, 1998). Fixations are brief periods of time in which the high-acuity fovea settles on most words. Saccades are high-velocity movements that step fixation from word to word through the text. Because eye movements are critical for efficient and effective reading, the nature of the processes that control these movements has been a focus of intense research (Engbert, Nuthmann, Richter \& Kliegl, 2005; Reichle, Pollatsek, Fisher \& Rayner, 1998).

A mechanism that may play an important role in eye movement control during reading but that has received relatively little consideration is inhibition of return (IOR). IOR is a decrease in processing efficiency for stimuli presented at recently attended locations. The classic demonstration of IOR is an increase in reaction time to a target presented at a previously attended location, as compared with a new location (Posner \& Cohen, 1984). IOR is a well-established phenomenon in the study of covert spatial attention and is observed across a wide variety of conditions (Klein, 2000).

Although the majority of paradigms used to study IOR concentrate on covert attention, IOR can also influence overt attention (eye movements). Oculomotor IOR (OIOR) is an increase in saccade latencies when the eyes move back to a previously fixated location, as compared with a new control location (Klein \& Hilchey, 2011). O-IOR, measured as an increase in fixation duration prior to a return saccade, has been reported in a variety of complex freeviewing tasks (Dodd, Van der Stigchel \& Hollingworth, 2009; Farrell, Ludwig, Ellis \& Gilchrist, 2010; Hooge \& 
Frens, 2000; Hooge, Over, van Wezel \& Frens, 2005; Klein \& MacInnes, 1999; MacInnes \& Klein, 2003; Ludwig, Farrell, Ellis \& Gilchrist, 2009; Smith \& Henderson, 2009, 2011a, b; Thomas et al., 2006).

The present study focused on O-IOR in reading. Reading is a complex perceptual and cognitive skill in which attention and eye movements must be coordinated to support language processing. The role of O-IOR in reading has been investigated in two previous studies (Rayner, Juhasz, Ashby \& Clifton, 2003; Weger \& Inhoff, 2006). Rayner et al. reported that fixation durations prior to regressions to previously fixated words are longer than those to previously skipped words. These results are consistent with O-IOR. However, Weger and Inhoff noted that regressions to previously fixated versus skipped words could support different processing functions, with these processing differences leading to differences in the fixation durations preceding forward movements and regressions. To circumvent this potential confound, Weger and Inhoff examined the amplitude of regressions in slow- and fast-buildup IOR groups of subjects (i.e., subjects who take longer to begin inhibiting return movements vs. those who do so almost immediately). They found that readers with fast-buildup IOR made larger regressions than those with slow-buildup IOR. They argued that IOR was applied to closer words, since those words were more recently fixated, which then prevented shorter regressions in the fast-buildup subjects. However, it is difficult to interpret spatial O-IOR (fixation probability) without evidence for temporal IOR (fixation duration), and it can be problematic to compare saccades of differing amplitudes when testing for IOR effects (Smith \& Henderson, 2009, 2011a, b) because saccade amplitude influences fixation duration directly (Rayner, 1998). Note that we use the term spatial O-IOR in this context not as a contrast to object-based IOR, but simply as a shorthand for IOR effects on fixation location versus fixation duration (temporal O-IOR).

The present study was designed to extend the research on O-IOR in reading. Subjects read paragraphs of text while their eye movements were recorded, and fixation durations prior to return saccades were compared with fixation durations prior to saccades to other control locations. Furthermore, O-IOR in reading was compared with a nonreading oculomotor scanning task that shared many of the eye movement characteristics of reading but eliminated meaning. In this mindless reading condition, subjects moved their eyes through stimuli that were arranged like text but that carried no lexical, syntactic, or semantic content (Nuthmann \& Engbert, 2009; Nuthmann, Engbert \& Kliegl, 2007; Vitu, O'Regan, Inhoff \& Topolski, 1995). Although there are important differences in eye movements during normal and mindless reading (Rayner \& Fisher, 1996), many eye movement characteristics in mindless reading are strikingly similar to those in normal reading (Nuthmann \& Engbert, 2009; Vitu et al., 1995). In the present version of mindless reading, each word was replaced with a geometric shape that maintained the overall visual structure of the text but eliminated meaning at all levels, including the level of letters (Fig. 1). This mindless reading condition provided a method for determining whether any O-IOR observed in reading was due to cognitive factors related to understanding the text or to oculomotor factors related to systematically moving the eyes.

In summary, the present study investigated O-IOR in reading in several novel ways. First, text reading was compared with mindless reading to determine the degree to which any O-IOR effects were due to oculomotor processing versus language processing. Second, subjects read a large amount of text, providing a significantly increased data set, as compared with past studies, over which to examine O-IOR effects. The increased data set allowed for the application of linear mixed effects (LMEs) modeling to the data analysis, permitting investigation of subtle effects in the data while simultaneously controlling for a number of variables that could potentially interact with O-IOR. Third, fixation location at the level of specific characters, in addition to whole words, was measured to investigate the spatial distribution and precision of IOR in reading. Fourth, the data were analyzed for both temporal and spatial components of O-IOR.

\section{Method}

Subjects Eight subjects from the University of South Carolina community, all native English speakers with 20/20 corrected or uncorrected vision, completed the experiment.

Apparatus Eye movements were recorded via an SR Research Eyelink 1000 eye tracker (spatial resolution of $0.01^{\circ}$ ) sampling at $1000 \mathrm{~Hz}$. Subjects were seated $69 \mathrm{~cm}$ away from a 20 -in. monitor so that approximately 3.5 characters subtended $1^{\circ}$ of visual angle. Head movements were minimized with chin- and headrests. Although viewing was binocular, eye movements were recorded from the right eye. The experiment was controlled with SR Research Experiment Builder software.

Materials Fifty-six paragraphs (40-80 words each) were taken from online news articles. Two different versions of each text were created: a normal reading version in which the text appeared on the screen in Courier New 16-point font and a mindless reading version in which the text was displayed in a custom font. This font transformed letters into block shapes (see Fig. 1). Both fonts were monospace, and all letters, words, and lines of text appeared in exactly the same location across fonts.

Reading condition (normal vs. mindless) was counterbalanced across two stimulus lists, and each subject saw 
Fig. 1 Example of the normal

Two goldfish, named Shaggy and Daphne, have become the smallest and reading and mindless reading conditions. Texts included a total of 1,415 unique words, 3 one-letter words, 30 two-letter words, 93 three-letter words, 197 four-letter words, 247 fiveletter words, 227 six-letter words, 218 seven-letter words, 159 eight-letter words, 110 nine-letter words, and 131 words ten letters or longer

hardiest survivors of the devastating February earthquake in

Christchurch, New Zealand. The fish spent four and a half months

trapped in their tank in the city's off-limits downtown without anyone

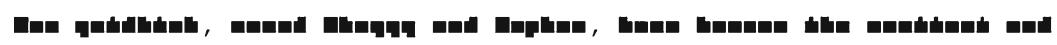

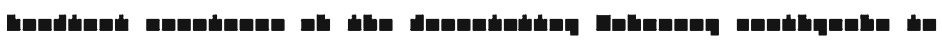

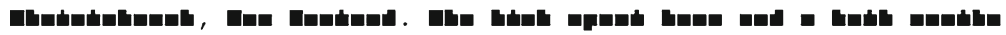

only one of the lists. Each subject saw 28 texts in the normal reading condition and 28 in the mindless reading condition, and no subject saw the same text twice.

Procedure Subjects were told that they would be reading short texts on a computer screen while their eye movements were recorded. Subjects were also told that some of the texts would appear with blocks in place of letters and that, in those cases, they should move their eyes as if they were reading. These are the standard instructions given in mindless reading experiments (Nuthmann et al., 2007; Vitu et al., 1995). Each trial involved the following sequence. The trial began with a gaze trigger, a black circle presented in the position of the first character in the text. Once a stable fixation was detected on the gaze trigger, the text was presented. The subject read the text and pressed a button to indicate when finished. Then a new gaze trigger appeared, and the next trial began. Texts were presented in a random order for each subject.

\section{Results}

Eye movements of interest were saccades that either moved the eyes to a previously fixated word (return saccades) or moved the eyes to a word that had not previously been fixated (nonreturn saccades). These saccades included regressions (backward movements) and forward movements that followed a regression. In every case, the eyes moved to a word that either had been previously fixated or had been skipped; eye movements to new text areas were not included. A saccade qualified as a return saccade if the eyes moved from the current word back to the word that had just been fixated, whether that word was in the forward or backward direction. Using Fig. 1 as an example, the following pattern would be classified as a regressive return fixation: a single fixation on electricity, a single fixation on power, followed by another fixation on electricity. A forward return fixation would involve fixations on power, electricity, and power, in that order. For nonreturn fixations, the third fixation was always a first fixation on a previously skipped word (e.g., devastating, earthquake, February). Thus, each set of eye movements consisted of three consecutive fixations. In order to explore temporal O-IOR effects, the duration of the second fixation (i.e., the one preceding the return or nonreturn saccade) was the primary dependent variable in the analyses below.

The criteria described above were the same as those used by Rayner et al. (2003). Consistent with Rayner et al., we also excluded fixations that were part of a series of two or 
more regressions. Also, fixations followed by extremely long (more than about 15 letters) and short (fewer than about 3 letters) saccades were eliminated to remove return sweeps, saccades resulting from severe comprehension difficulty, and short corrective saccades. The final data set included 3,058 saccades, 1,187 return saccades (732 forward), and 1,871 nonreturn saccades (1,334 forward). Chi-squared tests revealed a balanced distribution of saccades, except for a greater-than-expected number of forward nonreturn saccades in the normal text condition, $\chi^{2}=4.16, p<.05$. This suggests that there was no overall tendency to avoid returning to previously fixated words, except when subjects were returning to the right while reading.

The primary dependent variable was the duration of the fixation preceding the critical saccade. Fixation durations were log-transformed. Analyses were performed using linear mixed models in R (R Development Core Team, 2011), with subject and word as random effects. Only the final, best-fitted models are reported here. Random-effects structures were fitted using likelihood ratio tests and included all random intercepts (by subjects and by words) and slopes that contributed to the models. For the fixed-effects structures, only effects and interactions that were significant $(p<.05)$ were retained in the model. Factors were modeled using dummy coding. The $p$-values were obtained using Markov chain Monte Carlo sampling.

The primary model included three main predictors of fixation duration: saccade type (return/nonreturn), saccade direction (forward/regression), and reading condition (nor$\mathrm{mal} /$ mindless). The distributions of fixation durations as a function of these three variables are represented in Fig. 2.
This figure shows longer fixation times for mindless than for normal reading, a typical finding in the literature (Nuthmann et al., 2007; Vitu et al., 1995). The figure also shows longer fixation durations prior to return saccades than to nonreturn saccades, consistent with O-IOR.

The LME model confirmed these observations. Fixations were significantly shorter during normal reading than during mindless reading (coeff. $=-0.36, S E=$ 0.037), $t=-9.81, p<.001$. Additionally, fixations preceding regressions were shorter than fixations preceding forward saccades (coeff. $=-0.31, S E=0.07$ ) $, t=-4.5, p<$ .001 . These two predictors interacted (coeff. $=0.23, S E=$ $0.036), t=6.49, p<.001$, indicating that the difference between reading conditions was much smaller for fixations preceding regressions (effect size $=25 \mathrm{~ms}$ for regressions vs. $86 \mathrm{~ms}$ for forward saccades) and that the effect of saccade direction was much smaller in the normal reading condition ( $15 \mathrm{~ms}$ for normal vs. $76 \mathrm{~ms}$ for mindless reading). Most important, there was a significant effect of saccade type, with fixations preceding return saccades $31 \mathrm{~ms}$ longer that fixations preceding saccades to new words (coeff. $=0.1, S E=0.017$ ), $t=6.28, p<.001$. Saccade type did not interact with either saccade direction or reading condition, $t \mathrm{~s}<0.75$, indicating that the 31-ms IOR effect was independent of whether the eyes were moving backward or forward and whether subjects were engaged in text or mindless reading. Furthermore, in an analysis of the mindless reading condition alone, the 31-ms IOR effect was significant (coeff. $=0.11, S E=0.028), t=3.98, p<.001$.

To investigate whether fixation durations in the return saccade condition were inflated simply because saccades with greater amplitudes were generated in that condition, saccade
Fig. 2 Frequency distributions of fixation durations for regressive saccades (top) and forward saccades (bottom) for return and nonreturn saccades and for normal and mindless reading
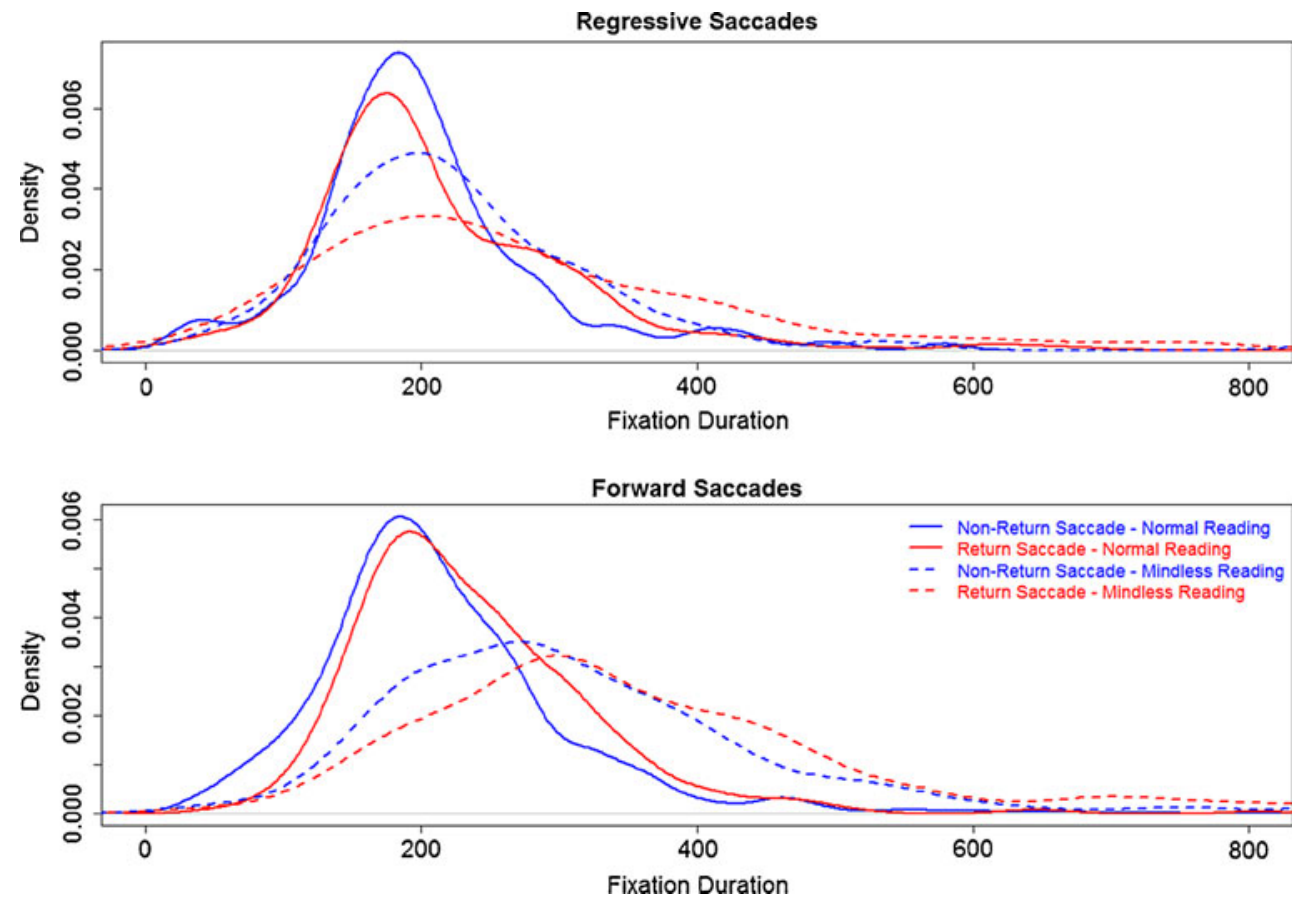
amplitude was also investigated. We separately analyzed saccade amplitudes that followed and that preceded the critical fixation. Both analyses revealed effects of saccade type and saccade direction, as well as a significant interaction of the two (following saccades, coeff. $=0.87, S E=0.066, t=13.19$, $p<.001$; preceding saccades, coeff. $=0.75, S E=0.063$, $t=11.77, p<.001)$. The interaction indicated that forward saccades had greater amplitude in the nonreturn condition, whereas the opposite was true for regressive saccades. The effect of reading condition was not significant, nor did it interact with the other factors, all $t \mathrm{~s}<1.8$. These analyses show that the O-IOR effects reported here are unlikely to be due to any increased time required to program or to recover from longer saccades.

Rayner et al. (2003) investigated the effect of return fixation location within a word to determine whether there was an increased IOR effect for return fixations that landed closer to the precise previous fixation location. They did not observe any such relationship and proposed that the IOR region in reading is defined over an entire word. We performed a similar analysis on the fixations that preceded return saccades only, including as a new predictor the linear distance in pixels between the previous fixation location within the target word and the return fixation on that word. Because only return saccades were analyzed, the initial and return fixations were always on the same word. This measure allows for a fine-grained analysis of the spatial distribution of IOR. The dependent variable was the duration of the fixation on the launch word that preceded the saccade to the previously fixated word. With this restricted data set, it was necessary to collapse fixations landing to the left or to the right of the original fixation location into one group, since there were not enough total fixations to permit separate treatment. This analysis replicated the results of the earlier analysis for both reading condition and saccade direction. In addition, we observed a significant negative relationship between fixation duration and the distance within the word between the locations of the previous and return fixations (coeff. $=-0.0016, S E=0.00054, t=-3, p<.001$; effect size $=58 \mathrm{~ms}$, or about $5.3 \mathrm{~ms}$ per letter), consistent with spatially precise O-IOR. Neither reading condition nor saccade direction interacted with distance, $t \mathrm{~s}<1.47$, and the effect of distance was present in the mindless reading data when analyzed separately (coeff. $=-0.0024, S E=0.001$ ), $t=-2.33, p<.05$.

Rayner et al. (2003) investigated the influence of the frequency of the launch word on the O-IOR effect. They had insufficient data to statistically test whether the frequency of this word modulated IOR, but the pattern of mean fixation times suggested no such relationship. We performed a similar analysis, excluding fixations in the mindless reading condition and on words for which no frequency was available. This data set included 1,477 fixations, 882 (563 forward) that preceded nonreturn saccades and 595 (340 forward) that preceded return saccades. As before, we observed significantly longer fixation durations before return saccades (coeff. $=0.071, S E=0.022$ ) $, t=3.26, p<.005$. We also observed a significant effect of log frequency (coeff. $=-0.0073$, $S E=0.0035), t=-2.09, p<.05$. These factors did not interact, $t<0.2$. These results are consistent with those reported by Rayner et al. in suggesting that O-IOR and lexical frequency may independently influence the duration of the current fixation.

The analyses reported above deal primarily with temporal O-IOR effects. To investigate whether O-IOR had any effect on the spatial distribution of fixations in the present study, differences in fixation locations between the previous fixation and the return fixation were measured. For these analyses, only return fixations were used. This data set included 1,239 return fixations, 765 in the normal and 474 in the mindless reading condition. To analyze the spatial distribution of these return fixations, they were binned (one-letter width) on the basis of their distance from the location of the previous fixation. Fixations that landed more than four letters away from the previous fixation were excluded. For each subject, the proportion of fixations in each bin was calculated to produce a fixation probability for that location. Probabilities for normal and mindless reading were calculated separately and are represented in Fig. 3.

An ANOVA with distance and reading condition as within-subjects factors was conducted to investigate the relationship between these two variables. There was a significant effect of distance, $F(8,56)=27.39, M S E=$ $0.002, p<.01$, but neither reading condition nor its interaction with distance were significant, $F \mathrm{~s}<0.66$. These results indicate that fixations were distributed similarly in the normal and mindless reading conditions. Any spatial IOR effect should manifest as decreased fixation probability at zero distance, so a linear regression analysis of the data points from the three central bins $(-1,0$, and +1$)$ was conducted to see whether there was any sign of nonlinearity around the zero point (Smith \& Henderson, 2011a). The analysis revealed a significant linear trend for distance (coeff. $=0.043, S E=0.01$ ), $t=4.43, p<.001$, but no effect of reading condition and no interaction $(p s>.11)$. Furthermore, the quadratic term for distance was not significant, nor was the interaction ( $p$ s $>.32$ ), indicating no deviation from linearity. Thus, there was no evidence that fixations returning to the same letter space as the previous fixation were significantly less probable than expected given the probability of fixations at the surrounding locations. There was also no evidence that the spatial distribution of fixations was different in the normal and mindless reading conditions. We emphasize that these results are based on acceptance of the null hypothesis and so should be treated with caution. 
Fig. 3 Mean fixation probabilities for return fixations in normal reading and mindless reading as a function of distance from the previous fixation. Error bars represent \pm 1 standard error

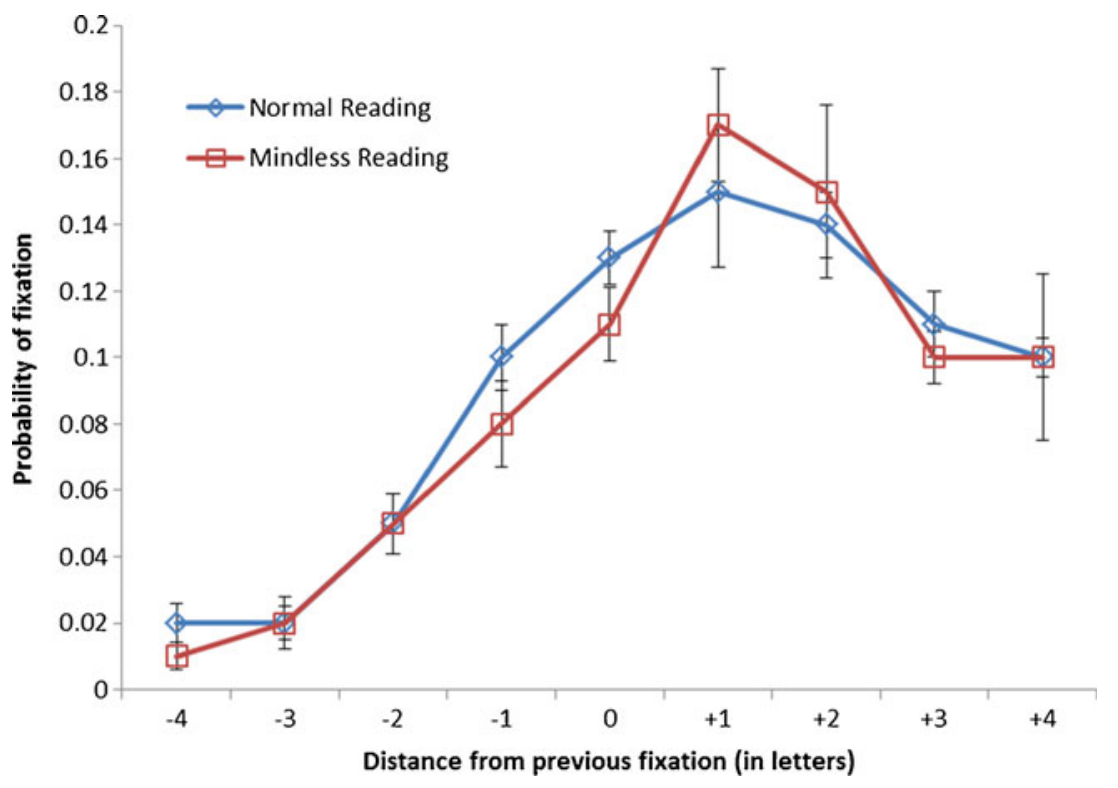

\section{Discussion}

The present study investigated O-IOR in reading. The central question we addressed was whether fixation durations are longer when the eyes are about to move back to a region of text that has just been fixated, as compared with a new region. To tease apart O-IOR effects due to cognitive processing related to text reading from more basic oculomotor effects related to systematic eye movements, O-IOR in normal text reading was compared with that in a mindless reading condition in which subjects moved their eyes through geometric shapes arranged like text but without meaning. We investigated IOR at the level of both the word and the level of the letter fixated within the word to shed light on the spatial distribution of O-IOR. The primary focus of the study was on temporal O-IOR effects. In a set of subsidiary analyses, we also examined whether the likelihood of looking back to a previously fixated location was reduced, as compared with other locations, a potential spatial consequence of O-IOR.

The results of the study were clear. Most important, fixations preceding saccades that returned to the word that was just fixated were $31 \mathrm{~ms}$ longer than fixations preceding saccades to new words. Furthermore, fixation durations were longest prior to a saccade that returned the eyes to the exact letter position that had previously been fixated, and fixation duration decreased as the distance between the previously fixated letter and the return fixation increased. This result suggests that O-IOR is applied to a specific character location and falls off in a gradient from there.

Importantly, the observed IOR effects were similar whether the subject was engaged in text reading or mindless reading. Furthermore, clear O-IOR effects were observed in the mindless reading condition alone. At the least, these results strongly suggest that O-IOR can be observed in a task that captures the oculomotor components of reading but that does not involve similar language or cognitive processes. The results are also consistent with the stronger hypothesis that the O-IOR effects observed in normal reading are entirely due to oculomotor programming related to moving the eyes from item to item, rather than to cognitive processes related to text processing during reading. However, this stronger conclusion must be treated with caution, given that it rests on acceptance of the null hypothesis regarding an IOR difference between the text reading and mindless reading conditions.

It has been suggested that O-IOR may have an influence on saccade targeting as well as saccade latency, with IOR producing a decrease in the probability that fixation will return to a location when that location has just been fixated (Klein \& Hilchey, 2011; Wang \& Klein, 2010). In this way, O-IOR could facilitate eye movement control by helping observers avoid reinspecting recently fixated locations (Klein, 1988; Klein \& MacInnes, 1999). Evidence concerning this hypothesis is currently mixed, with several reports of equal or greater refixation of previously fixated locations in visual search and scene viewing (Hooge et al., 2005; Smith \& Henderson, 2009, 2011a, 2011b). In the present study, little evidence for spatial consequences of O-IOR on fixation probability was observed. Although fewer fixations were found on previously fixated words than on other words when the eyes moved rightward, this pattern was not observed when the eyes moved leftward. Furthermore, no such evidence was found in the fine-grained analysis of fixation position focusing on letter position with a fixated word. These results are consistent with recent results from scene viewing and search suggesting that temporal O-IOR need not be accompanied by spatial O-IOR (Hooge et al., 2005; Smith \& Henderson, 2009, 2011a, b). 
Although reading is a relatively young skill from the perspective of evolution, it appears to draw on much older systems developed for controlling the dynamic allocation of attention and eye movements. The present study suggests that IOR is one such process that helps to determine how long the eyes remain fixated in a particular location during reading.

Author Note The research reported in this article was supported by a grant from the National Science Foundation (BCS-1151358) to John M. Henderson. Please address correspondence to John M. Henderson, Department of Psychology, 1512 Pendleton Street, Columbia, SC 29208.

\section{References}

Dodd, M. D., Van der Stigchel, S., \& Hollingworth, A. (2009). Novelty is not always the best policy: Inhibition of return and facilitation of return as a function of visual task. Psychological Science, 20, $333-339$.

Engbert, R., Nuthmann, A., Richter, E., \& Kliegl, R. (2005). SWIFT: A dynamical model of saccade generation during reading. Psychological Review, 112, 777-813.

Farrell, S., Ludwig, C. L., Ellis, L. A., \& Gilchrist, I. D. (2010). The influence of environmental statistics on inhibition of saccadic return. Proceedings of the National Academy of Sciences, 107, 929-934.

Hooge, I. T., \& Frens, M. A. (2000). Inhibition of saccade return (ISR): Spatial-temporal properties of saccade programming. Vision Research, 40, 3415-3426.

Hooge, I. T., Over, E. A., van Wezel, R. J., \& Frens, M. A. (2005). Inhibition of return is not a foraging facilitator in saccadic search and free viewing. Vision Research, 45, 1901-1908.

Klein, R. M. (1988). Inhibitory tagging system facilitates visual search. Nature, 334, 430-431.

Klein, R. M. (2000). Inhibition of return. Trends in Cognitive Sciences, 4, 138-146.

Klein, R. M., \& Hilchey, M. D. (2011). Oculomotor inhibition of return. In S. Liversedge, I. D. Gilchrist, \& S. Everling (Eds.), The Oxford handbook of eye movements (pp. 471-492). Oxford: Oxford University Press.

Klein, R. M., \& MacInnes, W. J. (1999). Inhibition of return is a foraging facilitator in visual search. Psychological Science, 10, $346-352$.
Ludwig, C. J. H., Farrell, S., Ellis, L. A., \& Gilchrist, I. D. (2009). The mechanism underlying inhibition of saccadic return. Cognitive Psychology, 59, 180-202.

MacInnes, W. J., \& Klein, R. M. (2003). Inhibition of return biases orienting during the search of complex scenes. The Scientific World Journal, 3, 75-86.

Nuthmann, A., \& Engbert, R. (2009). Mindless reading revisited: An analysis based on the SWIFT model of eye-movement control. Vision Research, 49, 322-336.

Nuthmann, A., Engbert, R., \& Kliegl, R. (2007). The IOVP effect in mindless reading: Experiment and modeling. Vision Research, 47, 990-1002.

Posner, M. I., \& Cohen, Y. (1984). Components of visual orienting. In H. Bouma \& D. Bouwhuis (Eds.), Attention and performance $X$ (pp. 531-556). London: Erlbaum.

R Development Core Team. (2011). R: A language and environment for statistical computing. Vienna: R Foundation for Statistical Computing. Retrieved from http://www.R-project.org/

Rayner, K. (1998). Eye movements in reading and information processing: 20 years of research. Psychological Bulletin, 124, 372422.

Rayner, K., \& Fisher, M. H. (1996). Mindless reading revisited: Eye movements during reading and scanning are different. Perception \& Psychophysics, 58, 734-747.

Rayner, K., Juhasz, B., Ashby, J., \& Clifton, C. (2003). Inhibition of saccade return in reading. Vision Research, 43, 1027-1034.

Reichle, E. D., Pollatsek, A., Fisher, D. L., \& Rayner, K. (1998). Toward a model of eye movement control in reading. Psychological Review, 105, 125-157.

Smith, T. J., \& Henderson, J. M. (2009). Facilitation of return during scene viewing. Visual Cognition, 17, 1083-1108.

Smith, T. J., \& Henderson, J. M. (2011a). Does oculomotor inhibition of return influence fixation probability during scene search? Attention, Perception, \& Psychophysics, 73, 2384-2398.

Smith, T. J., \& Henderson, J. M. (2011b). Looking back at Waldo: Oculomotor inhibition of return does not prevent return fixations. Journal of Vision, 11(1, Art. 3), 1-11.

Thomas, L. E., Ambinder, M. S., Hsieh, B., Levinthal, B., Crowell, J. A., Irwin, D. E., \& Wang, R. F. (2006). Fruitful visual search: Inhibition of return in a virtual foraging task. Psychonomic Bulletin \& Review, 13, 891-895.

Vitu, F., O'Regan, J. K., Inhoff, A. W., \& Topolski, R. (1995). Mindless reading: Eye-movement characteristics are similar in scanning letter strings and readings texts. Perception \& Psychophysics, 57, 352364.

Wang, Z., \& Klein, R. M. (2010). Searching for inhibition of return in visual search: A review. Vision Research, 50, 220-228.

Weger, U. W., \& Inhoff, A. W. (2006). Attention and eye movements in reading: Inhibition of return predicts the size of regressive saccades. Psychological Science, 17, 187-191. 